Aims To quantify, describe and compare non-prescribed agent (NPA) use by children with, and without, chronic illness

To explore families' experience and motivations for giving (or not) NPAs

Methods A questionnaire and semi-structured interview study using an explanatory sequential approach. Parents of children attending fracture, cystic fibrosis (CF), haemato-oncology, neurology and children's development clinics were recruited at first attendance during October-December 2011. Children attending fracture clinic with a chronic illness were re-categorised. Quantitative data were analysed using descriptive statistics and group-wise comparisons using chi-squared and unpaired t-tests. Qualitative data, collected from interviews until data saturation, were coded then thematically analysed independently by three researchers.

Results During the study period 664 families attended clinics, 295 completed a questionnaire (response rate 44.4\%). Response rates varied by clinic (fracture clinic 33.8\%, chronic clinics 57.8\%). $60.3 \%$ children were male with a mean age of 9.16 years (sd 4.87). Children with a chronic illness were younger $(p<0.001)$. Overall prevalence of NPA use was $37.3 \%$. Specific prevalences - 10\% fish oils, $16 \%$ vitamins, $15 \%$ probiotics, less commonly noted included homoeopathy and hyperbaric oxygen. Differences in NPA use between clinics were small $(33 \%-41.6 \%, p=0.21)$. NPA use was not associated with age, gender, or socioeconomic status. $46.3 \%$ parents reported having told their doctor about NPA use.

Sixteen parents completed an interview, representing all groups. Ten themes were identified, including the parental intention in providing (or withholding) NPAs. Themes describing why parents did, or did not, provide their children with NPAs included a wish to take an active role, trust (in health professionals, or in the remedy of choice) and accessibility of doctors or NPAs.

Conclusions This is the first UK-based study to compare NPA use between children with and without chronic illness, detailing parental motivators. These are complex but relate to intent, trust, and a wish to take an active role in their child's healthcare. The prevalence of NPA use in this study is similar to others. We found no association between NPA use and chronic illness.

\section{G124 BIG POISONS: WHAT INGESTED SUBSTANCES CAUSE SIGNIFICANT HARM TO YOUNG CHILDREN?}

doi:10.1136/archdischild-2013-304107.136

M Anderson. Department of Paediatric Medicine, Great North Children's Hospital, Newcastle upon Tyne, UK

Accidental poisoning of young children by ingestion of medicines and other substances is a relatively common reason for attendance at hospital in the UK, but infrequently results in significant harm. There is limited data regarding which substances cause major morbidity and mortality. This study aimed to identify which medicines and other substances are associated with death and serious illness in pre-school children.

The Office of National Statistics (ONS) was contacted and provided data relating to deaths in children due to poisoning between 2000 and 2010. The UK Paediatric Intensive Care Audit network (PICANet) was contacted and provided data relating to children admitted to intensive care as a result of poisoning between 2002 and 2012. Where possible, demographic data and the individual substance or class of drug was extracted.

21 deaths of children aged under 5 years occurred where the primary cause was poisoning due to medication between 2000 and 2010. Methadone was responsible for 9 of these deaths. 214 children (124 male:90 female) aged under 5 years were admitted to PICU between 2002 and 2012 as a result of poisoning with a median length of stay of 1 day (range $0.1-17.2$ days). The causative medicine was often not recorded and admissions due to therapeutic misadventure or idiosyncratic drug reactions were included within these numbers. The commonest medicines, where recorded, were benzodiazepines (24 admissions) and methadone (19 admissions). 4 children died ( 2 methadone, 1 benzodiazepine and 1 caustic alkali).

Existing databases are useful sources of data relating to poisoning in children but are limited, and it is not possible to differentiate easily between exploratory poisoning and therapeutic misadventure. However, the data available suggest that methadone is a significant danger to young children. It is vital that the presence of young children in the households of those on methadone programmes are taken into account, and alternatives are explored. More robust and systematic data collection might help identify other substances associated with significant risk and assist with targetted prevention measures.

\section{G125 PRIMARY AND SECONDARY CARE MULTI-SITE AUDIT OF NICE GUIDANCE ON URINARY TRACT INFECTIONS IN CHILDREN}

doi:10.1136/archdischild-2013-304107.137

${ }^{1} \mathrm{C}$ Platt, ${ }^{2} \mathrm{G}$ Gyorffy, ${ }^{3} \mathrm{~J}$ Dudley, ${ }^{4} \mathrm{~J}$ Banerjee, ${ }^{5} \mathrm{C}$ McNulty, ${ }^{6} \mathrm{~J}$ Larcombe, 'L Jadresic. ${ }^{1}$ General Paediatrics, Gloucestershire Hospitals NHS Foundation Trust, Gloucester, UK; ${ }^{2}$ General Paediatrics, Royal United Hospital, Bath, UK; ${ }^{P}$ Paediatric Nephrology, Bristol Children's Hospital, Bristol, UK; ' $E$ Emergency Medicine, University Hospitals of Leicester NHS Trust, Leicester, UK; ${ }^{5}$ Microbiology Department, Gloucestershire Hospitals NHS Foundation Trust, Gloucester, UK; ${ }^{6}$ Durham University, Centre for Integrated Healthcare Research, Durham, UK

Aims Children and families should expect to receive NICE compliant care before and after diagnosis of UTI and independently of where this diagnosis is made. We aim to highlight the challenges faced by different healthcare providers in the diagnosis and management of children with UTI by evaluating the implementation of key aspects of the 2007 NICE guidance across primary and secondary care. We report on the findings of this national multi-site health quality improvement partnership (HOIP) project.

Methods Retrospective audit of 900 consecutive children $<16$ years based in 4 areas across the UK. 4 secondary care providers and 10 GP centres representing a diverse patient group were involved. 7 criteria based on 3 key NICE priorities (improving diagnosis, improving clinical evaluation and providing guidance in follow up after diagnosis) were audited in total with a standard of $100 \%$ compliance set for each.

Results Through a manual search of health and microbiology records, data was collected over a period of 12 months in 2010 on 1018 children.

Testing for UTI within the recommended time frame in cases of unexplained fever in children $<3$ years was poor (35\% of cases in secondary care, $34 \%$ of cases in primary care). Only $52 \%$ of infants $<3$ months were treated appropriately with parenteral antibiotics. Recording of salient points in the history of children with suspected UTI was poor. Follow up investigations were organised appropriately in only $46 \%$ and $55 \%$ of cases from primary and secondary care respectively. Urinary dipstick testing as a first line strategy for diagnosis was performed relatively well in $67 \%$ and $75 \%$ of confirmed UTI cases in primary and secondary care respectively. $84 \%$ of infants $<3$ months with suspected UTI were referred appropriately to specialist services.

Conclusions This audit has highlighted areas of weakness in the management of children with UTI, in particular, accuracy of diagnosis in children $<3$ years and the recording of historical features that differentiate typical and atypical UTI. Our enhanced awareness of best practise will improve the outcomes for children with UTI in the long term. 